\title{
Metabolomics approach reveals effects of antihypertensives and lipid-lowering drugs on the human metabolism
}

\author{
Elisabeth Altmaier · Gisela Fobo • Margit Heier - Barbara Thorand • \\ Christine Meisinger · Werner Römisch-Margl • Melanie Waldenberger • \\ Christian Gieger - Thomas Illig • Jerzy Adamski - Karsten Suhre • \\ Gabi Kastenmüller
}

Received: 23 July 2013/Accepted: 28 April 2014/Published online: 10 May 2014

(C) The Author(s) 2014. This article is published with open access at Springerlink.com

\begin{abstract}
The mechanism of antihypertensive and lipidlowering drugs on the human organism is still not fully understood. New insights on the drugs' action can be provided by a metabolomics-driven approach, which offers a detailed view of the physiological state of an organism. Here, we report a metabolome-wide association study with 295 metabolites in human serum from 1,762 participants of the KORA F4 (Cooperative Health Research in the Region of Augsburg) study population. Our intent was to find variations of metabolite concentrations related to the intake of various drug classes and-based on the associations found-to generate new hypotheses about on-target as well
\end{abstract}

Electronic supplementary material The online version of this article (doi:10.1007/s10654-014-9910-7) contains supplementary material, which is available to authorized users.

E. Altmaier $(\square)$ · G. Fobo · W. Römisch-Margl · K. Suhre · G. Kastenmüller

Institute of Bioinformatics and Systems Biology, Helmholtz Zentrum München, German Research Center for Environmental Health, Ingolstädter Landstr. 1, 85764 Neuherberg, Germany

e-mail: elisabeth.altmaier@helmholtz-muenchen.de

E. Altmaier - C. Gieger

Institute of Genetic Epidemiology, Helmholtz Zentrum

München, German Research Center for Environmental Health,

Ingolstädter Landstr. 1, 85764 Neuherberg, Germany

M. Heier · B. Thorand - C. Meisinger

Institute of Epidemiology II, Helmholtz Zentrum München,

German Research Center for Environmental Health, Ingolstädter

Landstr. 1, 85764 Neuherberg, Germany

M. Waldenberger · T. Illig

Research Unit of Molecular Epidemiology, Helmholtz Zentrum

München, German Research Center for Environmental Health,

Ingolstädter Landstr. 1, 85764 Neuherberg, Germany as off-target effects of these drugs. In total, we found 41 significant associations for the drug classes investigated: For beta-blockers (11 associations), angiotensin-converting enzyme (ACE) inhibitors (four assoc.), diuretics (seven assoc.), statins (ten assoc.), and fibrates (nine assoc.) the top hits were pyroglutamine, phenylalanylphenylalanine, pseudouridine, 1-arachidonoylglycerophosphocholine, and 2-hydroxyisobutyrate, respectively. For beta-blockers we observed significant associations with metabolite concentrations that are indicative of drug side-effects, such as increased serotonin and decreased free fatty acid levels. Intake of ACE inhibitors and statins associated with metabolites that provide insight into the action of the drug itself on its target, such as an association of ACE inhibitors with des-Arg(9)-bradykinin and aspartylphenylalanine, a substrate and a product of the drug-inhibited ACE. The

\section{T. Illig}

Hannover Unified Biobank, Hannover Medical School, Carl-Neuberg-Str. 1, 30625 Hannover, Germany

J. Adamski

Institute of Experimental Genetics, Genome Analysis Center, Helmholtz Zentrum München, German Research Center for Environmental Health, Ingolstädter Landstr. 1,

85764 Neuherberg, Germany

\section{J. Adamski}

Institute of Experimental Genetics, Life and Food Science

Center Weihenstephan, Technische Universität München, 85354 Freising, Germany

\section{K. Suhre}

Department of Physiology and Biophysics, Weill Cornell Medical College in Qatar, Education City, Qatar Foundation, PO Box 24144, Doha, State of Qatar 
intake of statins which reduce blood cholesterol levels, resulted in changes in the concentration of metabolites of the biosynthesis as well as of the degradation of cholesterol. Fibrates showed the strongest association with 2-hydroxyisobutyrate which might be a breakdown product of fenofibrate and, thus, a possible marker for the degradation of this drug in the human organism. The analysis of diuretics showed a heterogeneous picture that is difficult to interpret. Taken together, our results provide a basis for a deeper functional understanding of the action and sideeffects of antihypertensive and lipid-lowering drugs in the general population.

Keywords Beta-blockers · Angiotensin-converting enzyme inhibitors · Diuretics · Statins · Fibrates · Metabolomics

\section{Introduction}

Hypertension is a risk factor for the development of atherosclerosis and cardiovascular diseases as well as for renal failure. Obesity and hyperlipidemia increase the risk of cardiovascular disease as well, as well as of diabetes mellitus type 2, gout and cognitive decline, including Alzheimer's disease. In addition to efforts to change lifestyle, antihypertensive drugs and lipid-lowering agents are prescribed. Due to the high prevalence of these risk factors, this medication ranks among the most frequently prescribed drugs in medical practice.

Beta-blockers are a heterogeneous class of antihypertensive drugs. Their main mode of action is the inhibition of noradrenaline and adrenaline $\beta$-adrenergic receptors, which diminishes the effect of the sympathetic nervous system on its target organ, especially on the smooth muscle tissue of the blood vessels and the heart. Most beta-blockers act on $\beta_{1^{-}}$, $\beta_{2}$ - and/or $\beta_{3}$-adrenergic receptors, while some beta-blockers of the newer generation show an additional vasodilatory effect by, for example, blocking $\alpha_{1}$-adrenergic receptors [1$3]$. Another important class of antihypertensive agents are angiotensin-I-converting enzyme (ACE) inhibitors. The ACE catalyzes the conversion of angiotensin-I to angiotensin-II which causes the muscles surrounding blood vessels to contract, thereby restricting the blood flow and increasing blood pressure. In addition, ACE cleaves and reduces the action of the vasodilator bradykinin and, thereby, increases the blood pressure. ACE inhibitors diminish the action of this enzyme and, thus, blood vessels dilate and blood pressure is reduced $[1,4]$. A drug therapy with diuretics has long been one of the most important treatments of hypertension and heart failure. However, today, many diuretics have found their main role in areas outside of hypertension. Diuretics inhibit the reabsorption of sodium in the renal tubules and their ability to alter long-term sodium balance induces important hemodynamic changes that result in a reduction in peripheral resistance and a decrease in blood pressure [5-7].

Two important classes of lipid-lowering drugs are statins and fibrates. Statins lower the concentration of cholesterol in the blood by reducing the cholesterol biosynthesis. The rate-limiting enzyme of this biosynthesis is the 3-hydroxy-3-methyl-glutaryl-CoA reductase (HMGCoA reductase; HMGCR), which is competitively inhibited by the statins $[8,9]$. In contrast, fibrates decrease the blood levels of fatty acids and triglycerides. They activate the peroxisome proliferator-activated receptor (PPAR), mainly PPAR- $\alpha$, which mediates the stimulation of the fatty acid $\beta$-oxidation [10-13].

In this study we used a pharmacometabolomic approach. Pharmacometabolomics aims to identify metabolic traits that offer insights into the intended as well as the unintentional effects of drugs on the human organism. The power of pharmacometabolomics was already shown in several studies [14-16]. One example is the study from Trupp et al. [14]. Examining the metabolic response to simvastatin treatment in 148 study participants they identified metabolites from multiple pathways not directly connected to the drug target which may explain the variation in therapeutic response.

For the identification of drug effects clinical drug testing is the standard approach. These trials are conducted under very controlled conditions and they are mainly focused on the objectives of the trials. The metabolomics approach for the analysis of medication effects in a big study population can provide a broader picture of the pathways affected by the respective drug by giving a snapshot of the physiological state of the organism. Using this kind of analysis, hypotheses about on-target as well as off-target effects can be generated, that reflect the drug action in the population and under everyday life conditions. A population based approach also allows the analysis of several drugs in the same study.

In this study, we used a mass spectrometry based nontargeted metabolomics approach to investigate the effect of antihypertensive and lipid-lowering agents. To this end, we analyzed the metabolic profiles of 1,762 participants from the KORA (Cooperative Health Research in the Region of Augsburg) study population for associations with the intake of these drugs (classification into cases/controls see Table 1).

\section{Materials and methods}

\section{Study population}

The research platform KORA conducts population-based surveys and subsequent follow-up studies in the fields of 
Table 1 Characteristics of the population related to antihypertensives and lipid-lowering drugs

\begin{tabular}{|c|c|c|c|c|c|}
\hline \multicolumn{3}{|l|}{ sives and lipid-lowe } & \multirow{4}{*}{$b$} & \multirow{2}{*}{\multicolumn{2}{|c|}{$\begin{array}{l}\text { Intake of ACE inhibitors } \\
\text { Mean (SD) or } \mathrm{n}(\%)\end{array}$}} \\
\hline \multirow{3}{*}{$a$} & \multirow{2}{*}{\multicolumn{2}{|c|}{$\begin{array}{l}\text { Intake of beta-blockers } \\
\text { Mean (SD) or } n(\%)\end{array}$}} & & & \\
\hline & & & & Yes $(n=282)$ & $\operatorname{No}(n=1480)$ \\
\hline & Yes $(n=403)$ & No $(\mathrm{n}=1,359)$ & & & \\
\hline Age & $65.43(7.45)$ & $59.46(8.66)$ & Yes & $261(14.8)$ & 394 (22.4) \\
\hline Gender & & & No & $21(1.2)$ & $1,086(61.6)$ \\
\hline Male & $201(11.4)$ & $655(37.2)$ & Intake of beta-blockers & & \\
\hline Female & $202(11.5)$ & $704(40.0)$ & Yes & $139(7.9)$ & $264(15.0)$ \\
\hline $\begin{array}{l}\text { HDL-cholesterol } \\
(\mathrm{mmol} / \mathrm{l})\end{array}$ & $1.34(0.35)$ & $1.49(0.38)$ & No & $143(8.1)$ & $1,216(69.0)$ \\
\hline $\begin{array}{l}\text { LDL-cholesterol } \\
(\mathrm{mmol} / \mathrm{l})\end{array}$ & $3.39(0.84)$ & $3.67(0.91)$ & $\begin{array}{l}\text { Intake of diuretics } \\
\text { Yes }\end{array}$ & $178(10.1)$ & $197(11.2)$ \\
\hline $\begin{array}{l}\text { Total cholesterol } \\
(\mathrm{mmol} / \mathrm{l})\end{array}$ & $5.43(0.95)$ & $5.81(1.01)$ & $\begin{array}{l}\text { No } \\
\text { Intake of statins }\end{array}$ & $104(5.9)$ & $1,283(72.8)$ \\
\hline Triglycerides (mmol/l) & $1.68(1.0)$ & $1.45(1.05)$ & Yes & $99(5.6)$ & $178(10.1)$ \\
\hline BMI kg/m² & $30.13(4.95)$ & $27.58(4.62)$ & No & $183(10.4)$ & $1,302(73.9)$ \\
\hline Diabetes mellitus & & & Intake of fibrates & & \\
\hline Yes & $69(3.9)$ & $92(5.2)$ & Yes & $3(0.2)$ & $8(0.5)$ \\
\hline No & $334(19.0)$ & $1,267(71.9)$ & No & $279(15.8)$ & $1,472(83.5)$ \\
\hline $\begin{array}{l}\text { Hypertension } \\
\text { Yes }\end{array}$ & $347(19.7)$ & $308(17.5)$ & $c$ & $\begin{array}{l}\text { Intake of diuret } \\
\text { Mean (SD) or } r\end{array}$ & \\
\hline $\begin{array}{l}\text { No } \\
\text { Intake of ACE inhibitors }\end{array}$ & $56(3.2)$ & $1,051(59.6)$ & & Yes $(n=375)$ & No $(n=1,387)$ \\
\hline Yes & $139(7.9)$ & $143(8.1)$ & Age & $65.90(7.44)$ & $59.45(8.59)$ \\
\hline No & $264(15.0)$ & $1,216(69.0)$ & Gender & & \\
\hline Intake of diuretics & & & Male & $195(11.1)$ & $661(37.5)$ \\
\hline Yes & $219(12.4)$ & $156(8.9)$ & Female & $180(10.2)$ & $726(41.2)$ \\
\hline No & $184(10.4)$ & $1,203(68.3)$ & HDL-cholesterol (mmol/l) & $1.37(0.33)$ & $1.48(0.38)$ \\
\hline Intake of statins & & & LDL-cholesterol (mmol/l) & $3.40(0.84)$ & $4.67(0.91)$ \\
\hline Yes & $144(8.2)$ & $133(7.5)$ & Total cholesterol $(\mathrm{mmol} / \mathrm{l})$ & $5.46(0.99)$ & $5.79(1.01)$ \\
\hline No & $259(14.7)$ & $1,226(69.6)$ & Triglycerides (mmol/l) & $1.66(0.98)$ & $1.47(1.05)$ \\
\hline Intake of fibrates & & & BMI kg/m² & $31.19(5.22)$ & $27.35(4.35)$ \\
\hline Yes & $5(0.3)$ & $6(0.3)$ & Diabetes mellitus & & \\
\hline No & $398(22.6)$ & $1,353(76.8)$ & Yes & $78(4.4)$ & $83(4.7)$ \\
\hline$b$ & Intake of ACE & bitors & No & $297(16.9)$ & $1,304(74.0)$ \\
\hline & Mean $(\mathrm{SD})$ or $\mathrm{n}$ & & Hypertension & & \\
\hline & Yes $(n=282)$ & No $(\mathrm{n}=1,480)$ & Yes & $348(19.8)$ & $307(17.4)$ \\
\hline & & & No & $27(1.5)$ & $1,080(61.3)$ \\
\hline Age & $65.97(7.77)$ & $59.84(8.60)$ & Intake of beta-blockers & & \\
\hline Gender & & & Yes & $219(12.4)$ & $184(10.4)$ \\
\hline Male & $165(9.4)$ & $691(39.2)$ & No & $156(8.9)$ & $1,203(68.3)$ \\
\hline Female & $117(6.6)$ & 789 (44.8) & Intake of ACE inhibitors & & \\
\hline HDL-cholesterol (mmol/l) & $1.34(0.33)$ & $1.48(0.38)$ & Yes & $178(10.1)$ & $104(5.9)$ \\
\hline LDL-cholesterol (mmol/l) & $3.42(0.89)$ & $3.65(0.90)$ & No & $197(11.2)$ & $1,283(72.8)$ \\
\hline Total cholesterol (mmol/l) & $5.47(0.99)$ & $5.77(1.01)$ & Intake of statins & & \\
\hline Triglycerides $(\mathrm{mmol} / \mathrm{l})$ & $1.69(1.01)$ & $1.47(1.05)$ & Yes & $124(7.0)$ & $153(8.7)$ \\
\hline $\mathrm{BMI} \mathrm{kg} / \mathrm{m}^{2}$ & $30.55(5.03)$ & $27.71(4.63)$ & No & $251(14.2)$ & $1,234(70.0)$ \\
\hline Diabetes mellitus & & & Intake of fibrates & & \\
\hline Yes & $70(4.0)$ & $91(5.2)$ & Yes & $3(0.2)$ & $8(0.5)$ \\
\hline No & $212(12.0)$ & $1,389(78.8)$ & No & $372(21.1)$ & $1,379(78.3)$ \\
\hline
\end{tabular}

Table 1 continued 
Table 1 continued

\begin{tabular}{|c|c|c|}
\hline \multirow[t]{2}{*}{$d$} & \multicolumn{2}{|c|}{$\begin{array}{l}\text { Intake of statins } \\
\text { Mean (SD) or } \mathrm{n}(\%)\end{array}$} \\
\hline & Yes $(n=277)$ & No $(\mathrm{n}=1,485)$ \\
\hline Age & $65.66(7.16)$ & $59.92(8.74)$ \\
\hline \multicolumn{3}{|l|}{ Gender } \\
\hline Male & $161(9.1)$ & $695(39.4)$ \\
\hline Female & $116(6.6)$ & $790(44.8)$ \\
\hline HDL-cholesterol (mmol/l) & $1.37(0.32)$ & $1.47(0.38)$ \\
\hline LDL-cholesterol (mmol/l) & $3.06(0.76)$ & $3.71(0.89)$ \\
\hline Total cholesterol (mmol/l) & $5.14(0.90)$ & $5.83(0.99)$ \\
\hline Triglycerides (mmol/l) & $1.69(1.03)$ & $1.47(1.04)$ \\
\hline BMI kg/m² & $29.30(4.85)$ & $27.95(4.77)$ \\
\hline \multicolumn{3}{|l|}{ Diabetes mellitus } \\
\hline Yes & $60(3.4)$ & $101(5.7)$ \\
\hline No & $217(12.3)$ & $1,384(78.5)$ \\
\hline \multicolumn{3}{|l|}{ Hypertension } \\
\hline Yes & $186(10.6)$ & 469 (26.6) \\
\hline No & $91(5.2)$ & $1,016(57.7)$ \\
\hline \multicolumn{3}{|l|}{ Intake of beta-blockers } \\
\hline Yes & $144(8.2)$ & $259(14.7)$ \\
\hline No & $133(7.5)$ & $1,226(69.6)$ \\
\hline \multicolumn{3}{|l|}{ Intake of ACE inhibitors } \\
\hline Yes & $99(5.6)$ & $183(10.4)$ \\
\hline No & $178(10.1)$ & $1,302(73.9)$ \\
\hline \multicolumn{3}{|l|}{ Intake of diuretics } \\
\hline Yes & $124(7.0)$ & $251(14.2)$ \\
\hline No & $153(8.7)$ & $1,234(70.0)$ \\
\hline \multicolumn{3}{|l|}{ Intake of fibrates } \\
\hline Yes & $0(0.0)$ & $11(0.6)$ \\
\hline No & $277(15.7)$ & $1,474(83.7)$ \\
\hline \multirow[t]{2}{*}{$e$} & \multicolumn{2}{|c|}{$\begin{array}{l}\text { Intake of fibrates } \\
\text { Mean (SD) or } n(\%)\end{array}$} \\
\hline & Yes $(n=11)$ & No $(\mathrm{n}=1,751)$ \\
\hline Age & $64.45(8.99)$ & $60.80(8.761)$ \\
\hline \multicolumn{3}{|l|}{ Gender } \\
\hline Male & $7(0.4)$ & $849(48.2)$ \\
\hline Female & $4(0.2)$ & $902(51.2)$ \\
\hline HDL-cholesterol (mmol/l) & $1.39(0.58)$ & $1.46(0.38)$ \\
\hline LDL-cholesterol (mmol/l) & $3.55(0.78)$ & $3.62(0.90)$ \\
\hline Total cholesterol (mmol/l) & $5.94(0.70)$ & $5.72(1.01)$ \\
\hline Triglycerides (mmol/l) & $2.81(2.82)$ & $1.50(1.02)$ \\
\hline BMI kg/m² & $28.40(3.63)$ & $28.16(4.82)$ \\
\hline \multicolumn{3}{|l|}{ Diabetes mellitus } \\
\hline Yes & $4(0.2)$ & $157(8.9)$ \\
\hline No & $7(0.4)$ & $1,594(90.5)$ \\
\hline \multicolumn{3}{|l|}{ Hypertension } \\
\hline Yes & $7(0.4)$ & $648(36.8)$ \\
\hline No & $4(0.2)$ & $1,103(62.6)$ \\
\hline
\end{tabular}

Table 1 continued

\begin{tabular}{lcr}
\hline \multirow{2}{*}{$e$} & \multicolumn{2}{l}{$\begin{array}{l}\text { Intake of fibrates } \\
\text { Mean (SD) or n }(\%)\end{array}$} \\
\cline { 2 - 3 } & Yes $(\mathrm{n}=11)$ & \multicolumn{1}{c}{ No $(\mathrm{n}=1,751)$} \\
\hline Intake of beta-blockers & $5(0.3)$ & $398(22.6)$ \\
Yes & $6(0.3)$ & $1,353(76.8)$ \\
No & & \\
Intake of ACE inhibitors & $3(0.2)$ & $279(15.8)$ \\
Yes & $8(0.5)$ & $1,472(83.5)$ \\
No & & \\
Intake of diuretics & $3(0.2)$ & $372(21.1)$ \\
Yes & $8(0.5)$ & $1,379(78.3)$ \\
No & & \\
Intake of statins & $0(0.0)$ & $277(15.7)$ \\
Yes & $11(0.6)$ & $1,474(83.7)$ \\
No & & \\
\hline
\end{tabular}

epidemiology, health care research, health economics and genetics. A multitude of different parameters is provided, including medical history, life style factors, and sociodemographic variables. The dataset analyzed here was taken from the F4 study, which was conducted in 2006-2008 as a follow-up of the fourth KORA survey (S4; 1999-2001). For this follow-up study F4, 3,080 of the 4,261 participants of the $\mathrm{S} 4$ were reexamined 7 years after baseline examination. From this group, the metabolic profiles of 1,768 participants, aged between 32 and 77 years were measured. In total, 1,762 of these study participants provided information about medication with beta-blockers, ACE inhibitors, diuretics, statins and/or fibrates. To determine the use of medication within the last 7 days before the examination an instrument for databaseassisted online collection of medication data (IDOM) was applied [17]. The drug classes were considered according to the recommendations of the German Hypertension Association [18]. The characteristics of the population related to these drug classes are shown in Table 1a-e. The usage of the agents within each drug class is given in the Supplemental Fig. 1. Informed consent was obtained from each study participant and all study protocols were approved by the local ethics committees.

Blood samples

For this study, we measured the metabolic profile in the blood serum of the study participants. To avoid variation due to circadian rhythm, blood was drawn in the morning between 8 and 10:30 am after overnight fasting (at least $8 \mathrm{~h}$ ). Medication was taken in the morning as usual. Material was drawn into serum gel tubes, gently inverted 
twice and followed by 30 min resting at room temperature $\left(18-25^{\circ} \mathrm{C}\right)$ to obtain complete coagulation. The material was then centrifuged for $10 \mathrm{~min}\left(2750 \mathrm{~g}\right.$ at $\left.15^{\circ} \mathrm{C}\right)$. Serum was aliquoted and stored at $4{ }^{\circ} \mathrm{C}$, after which it was deep frozen to $-80^{\circ} \mathrm{C}$ on the same day until analysis of the metabolites.

Metabolite profiling and metabolite spectrum

The metabolites were measured by the US-company Metabolon Inc., a commercial supplier of metabolic analyses. Their platform integrates the chemical analysis, including identification and relative quantification, data reduction, and quality assurance components of the process. Two separate ultrahigh performance liquid chromatography/ tandem mass spectrometry (UHPLC/MS/MS; positive and negative mode) injections and one gas chromatography/ mass spectrometry (GC/MS) injection were done on this platform.

The UHPLC/MS/MS platform utilized a Waters Acquity UPLC and a ThermoFisher LTQ mass spectrometer, while for GC/MS analysis a Thermo-Finnigan Trace DSQ MS was used.

A standard library containing retention time, molecular mass to charge ratio $(\mathrm{m} / \mathrm{z})$, preferred in-source fragments and adducts as well as their associated MS/MS spectra for all molecules in the library, subsequently enables to identify a multitude of metabolites in resulting MS/MS data. A more detailed description can be found in the Supplementary Material.

In a recent publication, Metabolon showed that their analytical platform is able to perform relative quantitative analysis of analytical data in a high-throughput mode and that it identifies a broad spectrum of molecules with a high degree of confidence [19]. Further details of the platform technology are described in Suhre et al. [20]. The measured panel includes 295 metabolites from many relevant classes such as amino acids, carbohydrates, cofactors and vitamins, acylcarnitines, glyceropospholipids, lipids, nucleotides, small peptides and xenobiotics.

This platform has been successfully used in several studies, e.g. for the analysis of the adult human plasma metabolome [21] and for the identification of sarcosine as a biomarker for prostate cancer [22].

\section{Statistical analysis}

The statistical analysis system R (http://www.r-project.org/) and SPSS for Windows (Version 19.0. Chicago: SPSS Inc.) were used for the statistical analysis.

The analytic approach applied by Metabolon is semiquantitative. This means that the added standards are mainly used for determining the retention time and not for the calculation of the metabolite concentrations. Thus, a relative intensity is measured and the measurement is sensitive to instrument parameters as well as fluctuations caused by maintenances like column change. These fluctuations are day dependent and the run day has to be considered for each individual. Thus, a run day normalization of the metabolic data was done: For each individual and each metabolite the data was first divided by the day median of the respective metabolite and then multiplied with the overall median of this metabolite.

To avoid false-positive associations, data points of metabolic traits that lay more than three standard deviations off the mean were excluded from further analysis. Metabolite concentrations were log-transformed since a test of normality showed that in most cases the log-transformed concentrations were closer to a normal distribution than the untransformed values.

For the identification of metabolites associated with medication use, a linear regression test was applied to model the association of each metabolite concentration and each of the five drug classes beta-blockers, ACE inhibitors, diuretics, statins and fibrates, respectively. As cofactors BMI, age, gender, HDL cholesterol, LDL cholesterol, total cholesterol, triglycerides, hypertension $(>160 / 95 \mathrm{mmHg}$ or known, medicated hypertension) and diabetes were chosen. To control for the effect of testing multiple hypotheses we used the Bonferroni correction. Thus, basing on a nominal level of 0.05 , we used $3.39 \times 10^{-5}[0.05 /(295$ metabolites * five drugs)] as an estimate of the significance level.

\section{Results}

For each drug class-beta-blockers, ACE inhibitors, diuretics, statins and fibrates-we analyzed the metabolic dataset to identify metabolites whose blood serum levels significantly differ between subjects taking and not taking the respective drug $\left(p\right.$ value smaller than $3.39 \times 10^{-5}$, the estimated significance level after correction for multiple testing-see "Statistical analysis"). The results of the linear regression are given in Table 2.

For patients who took beta-blockers we observed increased concentrations of pyroglutamine, homocitrulline, salicylate, and acylcarnitins in the blood serum. In contrast, serotonin, fatty acids and 3-hydroxybutyrate [FA(4:0-OH)] were decreased. Their $p$ values ranged between $1.9 \times 10^{-9}$ and $2.2 \times 10^{-5}$.

For the group of ACE inhibitors we identified four metabolites that significantly associated with the intake of these drugs. The $p$ values showed a broad range from $2.4 \times 10^{-80}$ up to $7.5 \times 10^{-13}$. While levels of HWESASXX and des-arg(9)-bradykinin were higher in case of medication with ACE inhibitors, we found lower levels of 


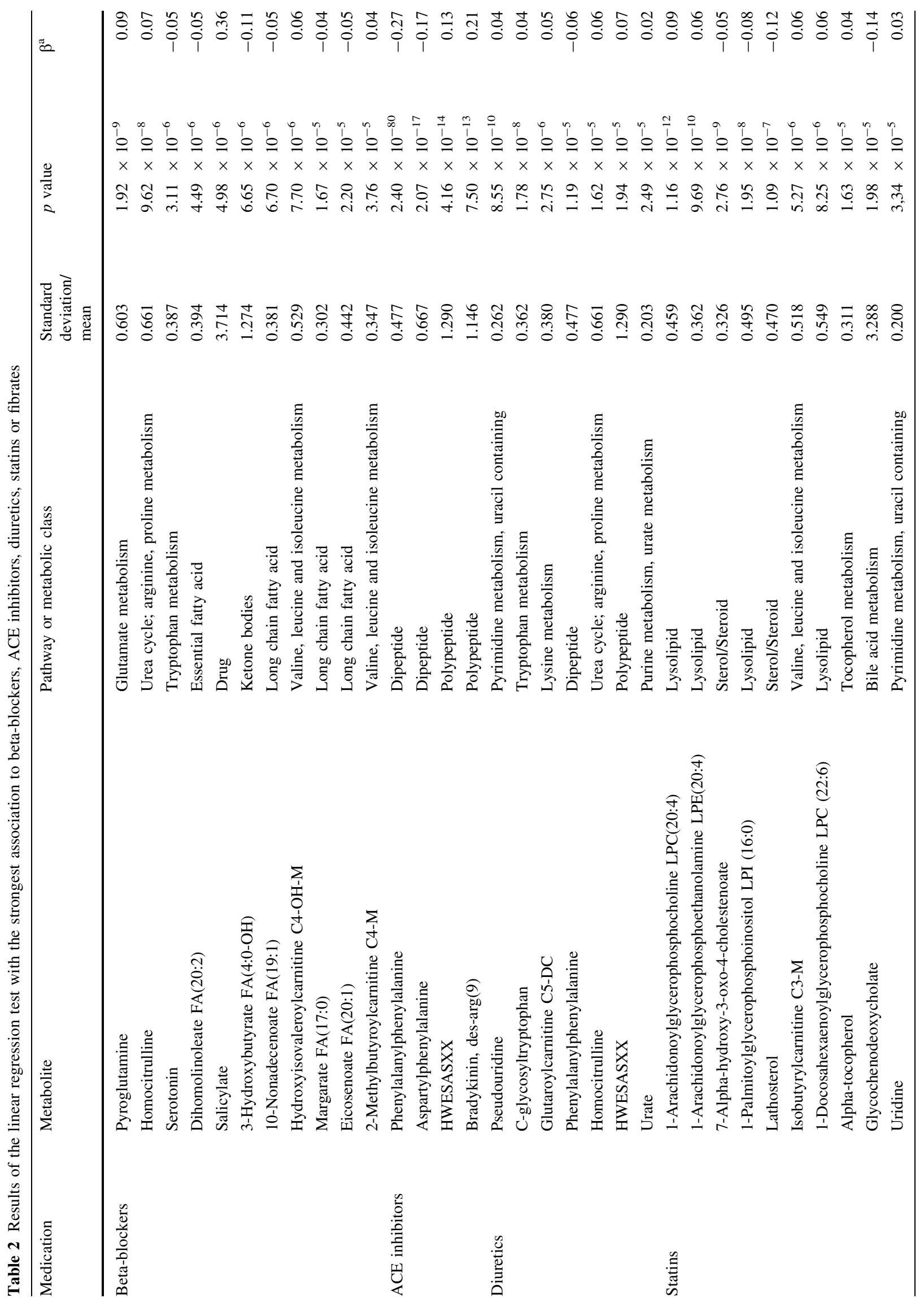


phenylalanylphenylalanine and aspartylphenylalanine. Diuretics showed associations with increased serum levels of pseudouridine, C-glycosyltryptophan, glutaroylcarnitine [C5-DC] and urate. Additional metabolites with a $p$ value smaller than $3.39 \times 10^{-5}$, namely homocitrulline, HWESASXX (both increased) and phenylalanylphenylalanine (decreased) were already found to associate with betablockers and ACE inhibitors, respectively.

To identify associations between lipid-lowering drugs and metabolites we analyzed the effects of statins as well as fibrates. For the statins the resulting metabolites with the lowest $p$ values were 1-arachidonoylglycerophosphocholine [LPC(20:4)], 1-arachidonoylglycerophosphoethanolamine [LPE(20:4)], isobutyrylcarnitine [C3-M], 1-docosahexaenoylglycerophosphocholine [LPC(22:6)], alpha-tocopherol, uridine (all increased), 7-alphahydroxy-3-oxo-4cholestenoate, 1-palmitoylglycerophosphoinositol [LPI(16:0)], lathosterol and glycochenodeoxycholate (all decreased). For fibrates most of the significant metabolites showed a positive association: 2-hydroxyisobutyrate [FA(3:0-OH-M)], 3-dehydrocarnitine, riboflavin, pantothenate, indolelactate, carnitine, pipecolate and uridine. Only for one of the resulting metabolites-pyroglutamine-a significant negative association was detected. Pyroglutamine was already observed to associate with the intake of betablockers. However, in contrast to the intake of fibrates, the association between the beta-blockers and the concentration of pyroglutamine was positive.

\section{Discussion}

In this study we analyzed the effect of antihypertensive drugs and lipid-lowering drugs on the human metabolism. To this end, 295 metabolites were measured in the serum of 1,762 participants of the population-based KORA F4 study. We found hypothesis-generating associations with metabolites for four different drugs, however, not for diuretics. The results of the linear regression are given in Table 2. In the following we will discuss the main results for each drug class.

Beta-blockers associate with decreased serotonin and free fatty acid levels

Beta-blockers diminish the effect of the sympathetic nervous system on its target organ mainly by inhibiting the action of noradrenaline and adrenaline on $\beta$-adrenergic receptors. Among the metabolites with the lowest $p$ values we found several fatty acids that were decreased with betablocker intake and some acylcarnitines that were increased. The increase in the concentration of acylcarnitines is also supported by the only nominally significant results. The 
decrease of free fatty acids agrees with the function of betablockers inhibiting the action of noradrenaline and adrenaline on $\beta$-adrenergic receptors. Since lipolysis is dependent on the action of these hormones, less triglycerides are broken down to free fatty acids [23, 24]. Vanhees et al. [25] also reported this effect for the beta-blocker bisoprolol which reduced the availability of plasma free fatty acids in healthy men.

Serotonin, a monoamine neurotransmitter, was significantly decreased in the blood of participants taking betablockers. Low plasma levels of serotonin were found in depressive patients $[26,27]$ and depression has been discussed as side-effect of beta-blockers [28]. Hence, the serotonin level variation might explain an adverse effect.

The metabolite with the strongest association to the intake of beta-blockers was pyroglutamine-a cyclic derivative of glutamine-that showed an increased concentration. In this context, it is noteworthy that an opposite behaviour, namely a decrease in the pyroglutamine concentration was associated with fibrate intake. However, further research on this metabolite is needed since too little is known so far about its physiological role.

In summary, our results for beta-blockers show associations that may indicate side effects of this medication.

\section{Direct action of ACE inhibitors on its target ACE}

The Angiotensin I-converting enzyme (ACE) cleaves not only angiotensin, but also several other polypeptides. One substrate of ACE is Cholecystokinin-8 (CCK-8) from which the dipeptide Asp-Phe-NH2 is cleaved [29]. In line with an inhibition of this process by ACE inhibitors, we found lower levels of aspartylphenylalanine (Asp-Phe) in users of ACE inhibitiors. Thus, one might expect that the substrate CCK-8 was increased in our patients on ACE inhibitors. Unfortunately, due to the used metabolite profiling technique CCK-8 was not in our metabolite panel and, therefore, we can only hypothesize. CCK-8 was shown to exert an antidiabetogenic action via increased insulin secretion [30]. Aguilar and Solomon suggested that ACE inhibitors may prevent the onset of diabetes [31] and Andraws and Brown could confirm this effect in a metaanalysis [32]. Thus, our observation of decreased levels of aspartylphenylalanine and the assumed elevated levels of CCK-8, suggest that this antidiabetogenic effect of ACE inhibitors is mediated by the inhibition of the cleavage of CCK-8. This hypothesis might be a starting point for further investigation.

The other dipeptide, phenylalanylphenylalanine, was also negatively associated with the intake of ACE inhibitors. It might be a product of the cleaving action of ACE as well, although the correspondent ACE substrate has not yet been identified. Candidate peptide substrates may now be searched for based on this information.

Another substrate of ACE is des- $\operatorname{Arg}(9)$-bradykininthe active metabolite of bradykinin - which causes blood vessels to dilate [33, 34]. Recent reports suggest an important role of lower bradykinin levels in the development of hypertension $[35,36]$. Thus, our observation of an increased blood level of des-Arg(9)-bradykinin under ACE inhibitors indicates the direct action of this drug and strengthens the suggested role of bradykinin in the treatment with ACE inhibitors [37].

Analysis of diuretics shows a heterogeneous picture

The results from the analysis of metabolic profiles of patients on diuretics are heterogeneous and difficult to interpret. An increased level of pseudouridine might indicate RNA degradation and cell turnover as well as the remodelling process in heart failure [38]. Our findings of an increase of C-glycosyltryptophan in patients on diuretics might be explained by the role of tryptophan. In liver cirrhosis the concentration of tryptophan is increased and tryptophan plays an important role in chronic kidney disease [39-41]. This is consistent with our observation of increased levels of glutaroylcarnitine [C5-DC], since glutaryl-CoA hydrogenase (GCDH) is essential in the degradation pathway of tryptophan [42]. A direct association between the metabolites pseudouridine and C-glycosyltryptophan and end stage renal disease (ESRD) was recently observed by Niewczas et al. [43]. They reported higher blood levels of these compounds in type 2 diabetes patients who progressed to ESRD within several years compared to diabetes patients without ESRD. Moreover, Yonemura et al. [44] observed a negative correlation between the C-glycosylated compound C-mannosyltryptophane and estimated glomerular filtration rate (eGFR; indicator of kidney function). An irreversible formation of homocitrulline (carbamoylation) is reported for end-stage renal disease and may explain the increased concentration of this metabolite [45].

Apart from their role as antihypertensives diuretics are used in cases of chronic kidney disease, heart failure and liver cirrhosis and, thus, we might see the metabolic traits of these diseases. Only the observed increased concentration of urate is a known side-effect of loop diuretics and thiazide [46].

Since more than $70 \%$ of the participants on diuretics used the agent hydrochlorothiazide (HCT) we repeated the analysis only with HCT-users. The two resulting metabolites with significant associations with HCT are pseudouridine and C-glycosyltryptophan. These metabolites were also the top hits in the analysis of the diuretics which suggests that the results for diuretics mainly reflect the results for HCT. 
Statins and their effects on the synthesis and degradation of cholesterol

Statins lower the concentration of cholesterol by inhibiting the HMG-CoA-reductase (HMGCR) which is the rate controlling enzyme of the biosynthesis of cholesterol. The levels of lathosterol and 7-alpha-hydroxy-3-oxo-4-cholestenoate were decreased in the blood from study participants treated with statins. Lathosterol is a precursor of cholesterol in the cholesterol biosynthesis, while 7-alphahydroxy-3-oxo-4-cholestenoate is a metabolite of cholesterol $[47,48]$. So these two metabolites show direct drug effects. The increased concentration of 1-arachidonoylglycerophosphocholine [LPC(20:4)] and 1-arachidonoylglycerophosphoethanolamine [LPE(20:4)] and the decreased level of 1-palmitoylglycerophosphoinositol [LPI(16:0)] agree with previous reports of an increase in arachidonic acid [FA(20:4)] synthesis and a decrease of palmitic acid [FA(16:0)] levels in persons on statins. This effect is assumed to be due to a statin-induced enhancement of delta five desaturase (FADS1) activity [49-51]. The benefit of the polyunsaturated fatty acid arachidonic acid is controversial. On the one hand arachidonic acid was reported as "beneficial in preventing and/or improving age- related declines in brain and cardiovascular system function" [52] as well as protective against oxidative stress in neurons [53]. On the other hand its role in inflammation is not fully clear, since anti- [54] as well as pro-inflammatory [55] effects have been described. A beneficial effect is achieved by the decrease of the 1-palmitoylglycerophosphoinositol [LPI(16:0)] carrying a saturated fatty acid which is considered as unhealthy.

In summary, for statins we observed mainly associations that are directly related to the action of this drug class.

Fibrates: degradation marker and increased carnitine levels

As a second group of lipid-lowering drugs, we analyzed the intake of fibrates for associations with metabolic traits. Though the group size for fibrate intake was quite small, associations with small $p$ values were observed due to the large effect size of the association. However, the possibility of a type I error cannot fully be excluded. The metabolite with the strongest association to fibrate intake was the upregulated 2-hydroxyisobutyrate [FA(3:0-OH-M)]. 2-hydroxyisobutyrate probably is a metabolite of fenofibrate. Liu et al. [56] propose a degradation of fenofibrate where
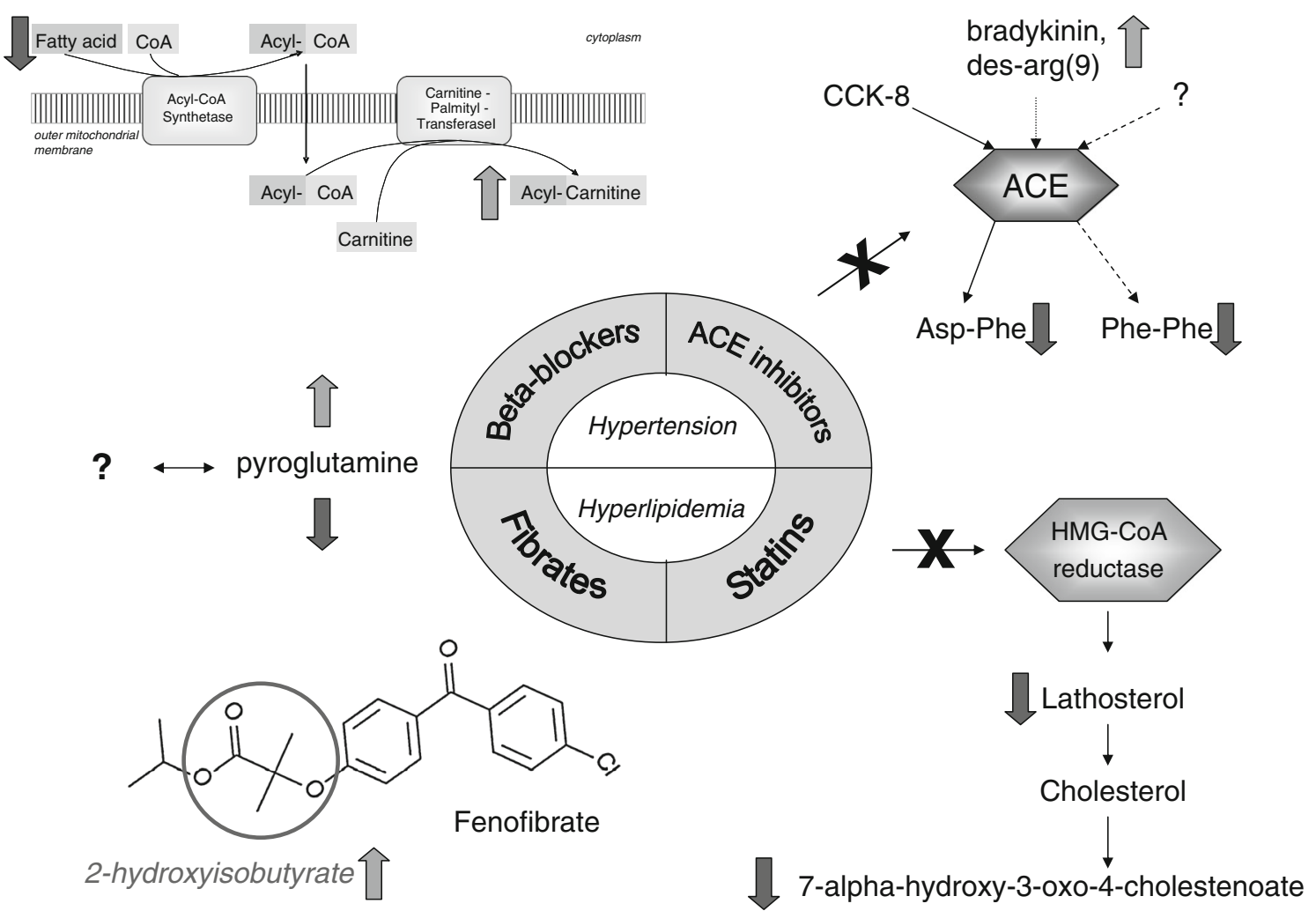

Fig. 1 Summary of the main drug-metabolite associations. Betablockers associate with an impaired lipid metabolism; the action of ACE inhibitors can be seen from associations with changed levels of substrate and products of ACE; statins associate with metabolites involved in the biosynthesis and degradation of cholesterol; fibrates associate with a hypothesized breakdown product of fenofibrate; pyroglutamine shows contrariwise associations with fibrates and betablockers 
reduced fenofibric acid is metabolized to reduced 4-chloro4 -hydroxybenzophenone. We presume that 2-hydroxyisobutyrate is released from reduced fenofibric acid during this reaction. If this is correct, 2-hydroxyisobutyrate might be used as a marker for the degradation of fenofibrate. In addition, 2-hydroxyisobutyrate was reported to be associated with prediagnostic gestational diabetes mellitus conditions [57] and obesity, and it might also be derived from the gut microbiome [58].

We also observed associations that might be related to side-effects of fibrates. 3-Dehydrocarnitine and free carnitine showed an increased concentration in the blood of patients on fibrates. This agrees with previous findings that fenofibrate increases the level of carnitine [59, 60]. The fact that 3-dehydrocarnitine is an intermediate in the carnitine biosynthesis may explain the increase of the blood concentration of this metabolite. Pyroglutamine was already the result with the lowest $p$ value in the betablockers analysis, but it also showed a strong association with the intake of fibrates. While with beta-blockers the association was positive, the association with fibrates was negative. The question if beta-blockers and fibrates influence the same metabolic phenomenon in opposite directions needs further investigation.

Our results show that indications for drug related metabolic changes can be identified in population studies although the participants are more influenced by their environment (e.g. xenobiotics, smoking, food, physical activity) and much less controlled than during a clinical drug testing, e.g. in phase 1. BMI, age, gender, HDL cholesterol, LDL cholesterol, total cholesterol, triglycerides, hypertension and diabetes are known to influence the here measured metabolic profile. To avoid seeing their disturbing influence in the results we used them as cofactors for our statistical analysis. In addition, we checked if any of the metabolites we found to be associated with beta-blockers, ACE inhibitors, statins or fibrates (not diuretics) shows an association with hypertension or the total blood cholesterol level in the non-medicated (no antihypertensives and no lipid-lowering drugs) participants. Except for one metabolite associated with the intake of statins (alpha-tocopherol), we found no such association, meaning that the associations observed for those drugs are mainly drug- and not disease-related. Also, a calculation considering for each drug the remaining four drugs as cofactors yielded the same significant associations.

\section{Limitations}

Our sampling population was composed of Caucasians of European ancestry, so these results cannot be generalized to other ethnicities - effects of genetic variation on metabolism needs to be considered [20,61, 62]. Because of the cross-sectional design of this study, we can only generate hypotheses based on associations-causal nature of the observed associations would require longitudinal data.

Major covariates, such as age, gender, BMI, HDL cholesterol, LDL cholesterol, total cholesterol, triglycerides, hypertension and diabetes were corrected for in the statistical analysis. However, in individual cases, interaction with other medication (e.g. with antiplatelet drugs or thyroid preparations) of the participants cannot be excluded.

To increase the statistical power the associations we reported in this paper were calculated for drug classes. It is not possible to trace the observed metabolic changes back to single pharmaceuticals. However, for each drug class specific associations could be clearly observed.

In summary (Fig. 1), for beta-blockers we observed associations possibly related to side-effects, including a decreased concentration of free fatty acids and a possible relation to depression via a decreased serotonin level. The intake of ACE inhibitors and statins associated with metabolites that reflect the direct action of the agent on its target. For fibrates we observed a possible breakdown product. Taken together, these results now provide a starting point for further functional research on the action and side-effects of these drugs.

Acknowledgments The authors are grateful for the commitment and involvement of all the study participants and for the work and dedication of the KORA Augsburg Study staff. The KORA research platform is financed by the Helmholtz Zentrum München, German Research Center for Environmental Health $(\mathrm{GmbH})$, which is funded by the German Federal Ministry of Education, Science, Research and Technology and by the State of Bavaria. This study was supported in part by a grant from the German Federal Ministry of Education and Research (BMBF) to the German Center for Diabetes Research (DZD e.V.), to the project "Systems Biology of Metabotypes (SysMBo)" and to the project "Greifswald Approach to Individualized Medicine (GANI_MED)" that was additionally financed by the Federal Government of Mecklenburg-Vorpommern. The research leading to these results has partly received funding from the European Union's Seventh Framework Programme (FP7-Health-F5-2012) under Grant agreement No 305280 (MIMOmics). K. S. is supported by "Biomedical Research Program" funds at Weill Cornell Medical College in Qatar, a program funded by the Qatar Foundation.

Conflict of interest The authors declare that they have no conflict of interest.

Open Access This article is distributed under the terms of the Creative Commons Attribution License which permits any use, distribution, and reproduction in any medium, provided the original author(s) and the source are credited.

\section{References}

1. Smith RE, Ashiya M. Antihypertensive therapies. Nat Rev Drug Discov. 2007;6(8):597-8. doi:10.1038/nrd2354. 
2. Baker JG, Hill SJ, Summers RJ. Evolution of beta-blockers: from anti-anginal drugs to ligand-directed signalling. Trends Pharmacol Sci. 2011;32(4):227-34. doi:10.1016/j.tips.2011.02.010.

3. Gorre F, Vandekerckhove H. Beta-blockers: focus on mechanism of action. Which beta-blocker, when and why? Acta Cardiol. 2010;65(5):565-70.

4. Li G-H, Le G-W, Shi Y-H, Shrestha S. Angiotensin I-converting enzyme inhibitory peptides derived from food proteins and their physiological and pharmacological effects. Nutr Res. 2004;24(7):469-86.

5. Ernst ME, Moser M. Use of diuretics in patients with hypertension. N Engl J Med. 2009;361(22):2153-64. doi:10.1056/ NEJMra0907219.

6. Group AOaCftACR. Major outcomes in high-risk hypertensive patients randomized to angiotensin-converting enzyme inhibitor or calcium channel blocker vs diuretic: the antihypertensive and lipid-lowering treatment to prevent heart attack trial (ALLHAT). JAMA. 2002;288(23):2981-97.

7. Ernst ME, Gordon JA. Diuretic therapy: key aspects in hypertension and renal disease. J Nephrol. 2010;23(5):487-93.

8. Krukemyer JJ, Talbert RL. Lovastatin: a new cholesterol-lowering agent. Pharmacotherapy. 1987;7(6):198-210.

9. Hebert PR, Gaziano JM, Chan KS, Hennekens CH. Cholesterol lowering with statin drugs, risk of stroke, and total mortality. An overview of randomized trials. JAMA. 1997;278(4):313-21.

10. Watts GF, Dimmitt SB. Fibrates, dyslipoproteinaemia and cardiovascular disease. Curr Opin Lipidol. 1999;10(6):561-74.

11. Ozasa $\mathrm{H}$, Miyazawa S, Furuta $\mathrm{S}$, Osumi $\mathrm{T}$, Hashimoto $\mathrm{T}$. Induction of peroxisomal beta-oxidation enzymes in primary cultured rat hepatocytes by clofibric acid. J Biochem. 1985;97(5): 1273-8.

12. Reddy JK, Goel SK, Nemali MR, Carrino JJ, Laffler TG, Reddy $\mathrm{MK}$, et al. Transcription regulation of peroxisomal fatty acylCoA oxidase and enoyl-CoA hydratase/3-hydroxyacyl-CoA dehydrogenase in rat liver by peroxisome proliferators. Proc Natl Acad Sci USA. 1986;83(6):1747-51.

13. Pahan K. Lipid-lowering drugs. Cell Mol Life Sci. 2006;63(10): 1165-78. doi:10.1007/s00018-005-5406-7.

14. Trupp M, Zhu H, Wikoff WR, Baillie RA, Zeng ZB, Karp PD, et al. Metabolomics reveals amino acids contribute to variation in response to simvastatin treatment. PLoS ONE. 2012;7(7):e38386. doi:10.1371/journal.pone.0038386.

15. Wikoff WR, Frye RF, Zhu H, Gong Y, Boyle S, Churchill E, et al. Pharmacometabolomics reveals racial differences in response to atenolol treatment. PLoS ONE. 2013;8(3):e57639. doi:10.1371/ journal.pone.0057639.

16. Corona G, Rizzolio F, Giordano A, Toffoli G. Pharmaco-metabolomics: an emerging "omics" tool for the personalization of anticancer treatments and identification of new valuable therapeutic targets. J Cell Physiol. 2012;227(7):2827-31. doi:10.1002/ jcp. 24003.

17. Mühlberger N, Behrend C, Stark R. Datenbankgestützte OnlineErfassung von Arzneimitteln im Rahmen gesundheitswissenschaftlicher Studien - Erfahrungen mit der IDOM-Software. Informatik Biometrie Epidemiologie Medizin Biologie. 2003;34:601-11.

18. Deutsche H. Empfehlung zur Hochdruckbehandlung. 19 Auflage. 2007.

19. Evans AM, DeHaven CD, Barrett T, Mitchell M, Milgram E. Integrated, nontargeted ultrahigh performance liquid chromatography/electrospray ionization tandem mass spectrometry platform for the identification and relative quantification of the smallmolecule complement of biological systems. Anal Chem. 2009;81(16):6656-67. doi:10.1021/ac901536h.

20. Suhre K, Shin SY, Petersen AK, Mohney RP, Meredith D, Wagele B, et al. Human metabolic individuality in biomedical and pharmaceutical research. Nature. 2011;477(7362):54-60. doi:10.1038/nature 10354

21. Lawton KA, Berger A, Mitchell M, Milgram KE, Evans AM, Guo L, et al. Analysis of the adult human plasma metabolome. Pharmacogenomics. 2008;9(4):383-97. doi:10.2217/14622416.9. 4.383.

22. Sreekumar A, Poisson LM, Rajendiran TM, Khan AP, Cao Q, Yu $\mathrm{J}$, et al. Metabolomic profiles delineate potential role for sarcosine in prostate cancer progression. Nature. 2009;457(7231): 910-4. doi:10.1038/nature07762.

23. Lafontan M, Berlan M. Fat cell adrenergic receptors and the control of white and brown fat cell function. J Lipid Res. 1993;34(7):1057-91.

24. Millet L, Barbe P, Lafontan M, Berlan M, Galitzky J. Catecholamine effects on lipolysis and blood flow in human abdominal and femoral adipose tissue. J Appl Physiol. 1998;85(1): $181-8$.

25. Vanhees L, Defoor JG, Schepers D, Lijnen P, Peeters BY, Lacante $\mathrm{PH}$, et al. Effect of bisoprolol and atenolol on endurance exercise capacity in healthy men. J Hypertens. 2000;18(1):35-43.

26. Lacasse JR, Leo J. Serotonin and depression: a disconnect between the advertisements and the scientific literature. PLoS Med. 2005;2(12):e392. doi:10.1371/journal.pmed.0020392.

27. Bakkaloğlu B, Yabanoğlu S, Özyüksel BR, Uçar G, Ertuğrul A, Demir B, et al. Platelet and plasma serotonin levels and platelet monoamine oxidase activity in patients with major depression: effects of sertraline treatment. Turk J Biochem. 2008;33(3): 97-103.

28. Verbeek DE, van Riezen J, de Boer RA, van Melle JP, de Jonge P. A review on the putative association between beta-blockers and depression. Heart Fail Clin. 2011;7(1):89-99. doi:10.1016/j. hfc.2010.08.006.

29. Dubreuil P, Fulcrand P, Rodriguez M, Fulcrand H, Laur J, Martinez J. Novel activity of angiotensin-converting enzyme. Hydrolysis of cholecystokinin and gastrin analogues with release of the amidated C-terminal dipeptide. Biochem J. 1989;262(1): $125-30$.

30. Ahren B, Holst JJ, Efendic S. Antidiabetogenic action of cholecystokinin- 8 in type 2 diabetes. J Clin Endocrinol Metab. 2000;85(3):1043-8.

31. Aguilar D, Solomon SD. ACE inhibitors and angiotensin receptor antagonists and the incidence of new-onset diabetes mellitus: an emerging theme. Drugs. 2006;66(9):1169-77.

32. Andraws R, Brown DL. Effect of inhibition of the renin-angiotensin system on development of type 2 diabetes mellitus (metaanalysis of randomized trials). Am J Cardiol. 2007;99(7): 1006-12. doi:10.1016/j.amjcard.2006.10.068.

33. Skidgel RA, Erdos EG. The broad substrate specificity of human angiotensin I converting enzyme. Clin Exp Hypertens A. 1987;9(2-3):243-59.

34. Cyr M, Lepage Y, Blais C Jr, Gervais N, Cugno M, Rouleau JL, et al. Bradykinin and des-Arg(9)-bradykinin metabolic pathways and kinetics of activation of human plasma. Am J Physiol Heart Circ Physiol. 2001;281(1):H275-83.

35. Sharma JN. Hypertension and the bradykinin system. Curr Hypertens Rep. 2009;11(3):178-81.

36. Katori M, Majima M. A missing link between a high salt intake and blood pressure increase. J Pharmacol Sci. 2006;100(5): 370-90.

37. Tom B, Dendorfer A, de Vries R, Saxena PR, Jan Danser AH. Bradykinin potentiation by ACE inhibitors: a matter of metabolism. Br J Pharmacol. 2002;137(2):276-84. doi:10.1038/sj.bjp.0704862.

38. Dunn W, Broadhurst D, Deepak S, Buch M, McDowell G, Spasic I, et al. Serum metabolomics reveals many novel metabolic markers of heart failure, including pseudouridine and 2-oxoglutarate. Metabolomics. 2007;3(4):413-26. 
39. Roessle M, Herz R, Klein B, Gerok W. Tryptophan-Metabolismus bei Lebererkrankungen: Eine pharmakokinetische und enzymatische Untersuchung. J Mol Med. 1986;64(13):590-4.

40. Schulman G. A nexus of progression of chronic kidney disease: charcoal, tryptophan and profibrotic cytokines. Blood Purif. 2006;24(1):143-8. doi:10.1159/000089451.

41. Niwa T. Uremic toxicity of indoxyl sulfate. Nagoya J Med Sci. 2010;72(1-2):1-11.

42. Monavari AA, Naughten ER. Prevention of cerebral palsy in glutaric aciduria type 1 by dietary management. Arch Dis Child. 2000;82(1):67-70.

43. Niewczas MA, Sirich TL, Mathew AV, Skupien J, Mohney RP, Warram $\mathrm{JH}$, et al. Uremic solutes and risk of end-stage renal disease in type 2 diabetes: metabolomic study. Kidney Int. 2014; doi:10.1038/ki.2013.497.

44. Yonemura K, Takahira R, Yonekawa O, Wada N, Hishida A. The diagnostic value of serum concentrations of 2-(alpha-mannopyranosyl)-L-tryptophan for normal renal function. Kidney Int. 2004;65(4):1395-9. doi:10.1111/j.1523-1755.2004.00521.x.

45. Kraus LM, Gaber L, Handorf CR, Marti HP, Kraus AP Jr. Carbamoylation of glomerular and tubular proteins in patients with kidney failure: a potential mechanism of ongoing renal damage. Swiss Med Wkly. 2001;131(11-12):139-44.

46. DeMarco MAM, Maynard JW, Baer AN, Gelber AC, Young JH, Alonso A, et al. Diuretic use, increased serum urate levels, and risk of incident gout in a population-based study of adults with hypertension: the Atherosclerosis Risk in Communities cohort study. Arthritis Rheum. 2012;64(1):121-9. doi:10.1002/Art. 33315.

47. Meaney S, Babiker A, Lutjohann D, Diczfalusy U, Axelson M, Bjorkhem I. On the origin of the cholestenoic acids in human circulation. Steroids. 2003;68(7-8):595-601.

48. Kempen HJ, Glatz JF, Gevers Leuven JA, van der Voort HA, Katan MB. Serum lathosterol concentration is an indicator of whole-body cholesterol synthesis in humans. J Lipid Res. 1988;29(9):1149-55.

49. Rise P, Pazzucconi F, Sirtori CR, Galli C. Statins enhance arachidonic acid synthesis in hypercholesterolemic patients. Nutr Metab Cardiovasc Dis. 2001;11(2):88-94.

50. Jula A, Marniemi J, Ronnemaa T, Virtanen A, Huupponen R. Effects of diet and simvastatin on fatty acid composition in hypercholesterolemic men: a randomized controlled trial. Arterioscler Thromb Vasc Biol. 2005;25(9):1952-9. doi:10.1161/01. ATV.0000177812.84927.fa.

51. Bellini MJ, Polo MP, de Alaniz MJ, de Bravo MG. Effect of simvastatin on the uptake and metabolic conversion of palmitic, dihomo-gamma-linoleic and alpha-linolenic acids in A549 cells. Prostaglandins Leukot Essent Fatty Acids. 2003;69(5):351-7.
52. Kiso Y. Pharmacology in health foods: effects of arachidonic acid and docosahexaenoic acid on the age-related decline in brain and cardiovascular system function. J Pharmacol Sci. 2011;115(4):471-5.

53. Wang ZJ, Liang CL, Li GM, Yu CY, Yin M. Neuroprotective effects of arachidonic acid against oxidative stress on rat hippocampal slices. Chem-Biol Interact. 2006;163(3):207-17. doi:10. 1016/j.cbi.2006.08.005.

54. Ferrucci L, Cherubini A, Bandinelli S, Bartali B, Corsi A, Lauretani $\mathrm{F}$, et al. Relationship of plasma polyunsaturated fatty acids to circulating inflammatory markers. J Clin Endocrinol Metab. 2006;91(2):439-46. doi:10.1210/jc.2005-1303.

55. Sears B, Ricordi C. Role of fatty acids and polyphenols in inflammatory gene transcription and their impact on obesity, metabolic syndrome and diabetes. Eur Rev Med Pharmacol Sci. 2012;16(9):1137-54.

56. Liu A, Patterson AD, Yang Z, Zhang X, Liu W, Qiu F, et al. Fenofibrate metabolism in the cynomolgus monkey using ultraperformance liquid chromatography-quadrupole time-of-flight mass spectrometry-based metabolomics. Drug Metab Dispos. 2009;37(6):1157-63. doi:10.1124/dmd.108.025817.

57. Diaz SO, Pinto J, Graca G, Duarte IF, Barros AS, Galhano E, et al. Metabolic biomarkers of prenatal disorders: an exploratory NMR metabonomics study of second trimester maternal urine and blood plasma. J Proteome Res. 2011;10(8):3732-42. doi:10.1021/ pr200352m.

58. Calvani R, Miccheli A, Capuani G, Tomassini Miccheli A, Puccetti C, Delfini M, et al. Gut microbiome-derived metabolites characterize a peculiar obese urinary metabotype. Int $\mathrm{J}$ Obes. 2010;34(6):1095-8. doi:10.1038/ijo.2010.44.

59. Gerondaes P, Alberti KG, Agius L. Interactions of inhibitors of carnitine palmitoyltransferase I and fibrates in cultured hepatocytes. Biochem J. 1988;253(1):169-73.

60. Tsoko M, Beauseigneur F, Gresti J, Demarquoy J, Clouet P. Hypolipidaemic effects of fenofibrate are not altered by mildronate-mediated normalization of carnitine concentration in rat liver. Biochimie. 1998;80(11):943-8.

61. Illig T, Gieger C, Zhai G, Romisch-Margl W, Wang-Sattler R, Prehn C, et al. A genome-wide perspective of genetic variation in human metabolism. Nat Genet. 2010;42(2):137-41. doi:10.1038/ ng.507.

62. Gieger C, Geistlinger L, Altmaier E, Hrabé de Angelis M, Kronenberg F, Meitinger T, et al. Genetics meets metabolomics: a genome-wide association study of metabolite profiles in human serum. PLoS Genet. 2008;4(11):e1000282. doi:10.1371/journal. pgen. 1000282. 